\title{
A Device for Measuring 3-Phase Alternating Current at a Distance
}

\author{
Seppo Mäkinen \\ School of Technology, Vaasa University of Applied Sciences, Vaasa, Finland \\ Email: seppo.makinen@vamk.fi
}

Received 10 July 2015; accepted 24 August 2015; published 27 August 2015

Copyright (C) 2015 by author and Scientific Research Publishing Inc.

This work is licensed under the Creative Commons Attribution International License (CC BY). http://creativecommons.org/licenses/by/4.0/

c) (i) Open Access

\begin{abstract}
We have made a preliminary study on a possibility to build a device, which could measure threephase alternating current at a distance. This report gives a brief look at the theoretical background related with such a device and derives several mathematical results associated with the theory and the structure of the device. Our results suggest that such a device can be built, assuming the distances between the three current-carrying wires and the two sensors of the device are known accurately enough. There are ways to measure these distances, but this article does not concentrate on them.
\end{abstract}

\section{Keywords}

\section{Electromagnetic Induction, 3-Phase Alternating Current, Ammeter}

\section{Introduction}

For energy companies, it is important to know if the transmission lines are working as they should or not. Further, they need this information all the time. They also want to know about the quality of the electric current flowing in the lines. Naturally, they have meters to find out the characteristics of the alternating current in their interest, but installation of a meter to a transmission line requires cutting off the current in the line. This is timeconsuming and requires more work than the mere installation.

In our recent paper, we discussed the use of a toroidal coil in measuring alternating current flowing in one wire [1]. We wanted to increase the challenge, and decided to consider three-phase alternating current flowing in three wires. In addition, we wanted to consider a meter, which could measure all three currents as functions of time- and so that such a meter could be installed to the transmission line safely without cutting off the current in the line. We decided to concentrate on the typical $20 \mathrm{kV}$ transmission line, which is shown in Figure 1.

Since the geometry shown in Figure 1 is not the only possible one, we need to build our model so that the 


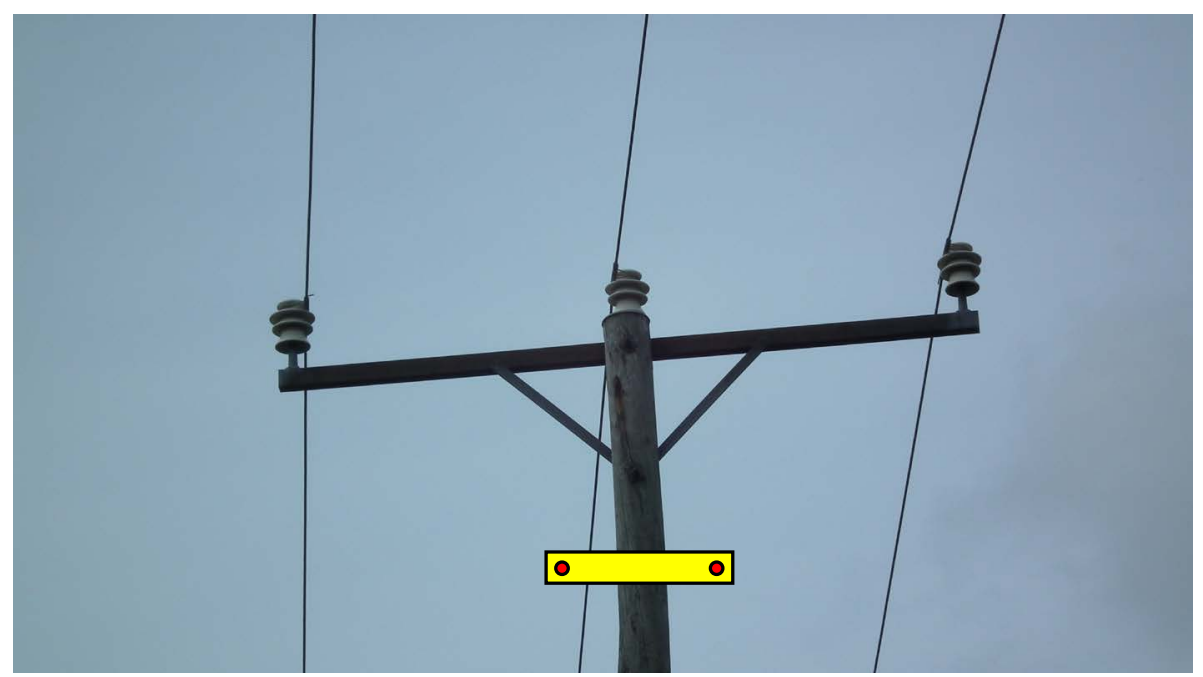

Figure 1. A typical $20 \mathrm{kV}$ transmission line with three current-conducting wires.

locations of the three wires are not restricted in any way. This means that we need to introduce a system of coordinates fixed to the device, relative to which the locations of the wires are specified. An illustration of the device is added to Figure 1.

\section{The Numerical Model}

Let us consider three straight and parallel conductors, which carry sinusoidally oscillating currents. We assume that the positions of the wires are not known, and that the phase differences between the three currents are unknown, as well. Further, we assume that the currents may have different amplitudes, and that we do not know these amplitudes beforehand. Thus, the only thing we do know about the case is that the currents are sinusoidal in shape, and that they oscillate at a given frequency. In addition, our analysis is built so that the number of currents is known to be three.

Our task is to find the three currents as functions of time. This means that we have altogether 9 unknowns (if the phase angles $\varphi_{1}, \varphi_{2}$ and $\varphi_{3}$ are ignored in the analysis): the $x$ and the $y$ coordinates of each wire, and the amplitudes of the three currents. For an unequivocal solution, we would thus need at least 9 equations in terms of these 9 variables. Solving such a set of equations seems too time-consuming, and we see that the only practical way to find the currents is to start with more initial information about the system.

In our opinion, the easiest way to obtain more information about the system is to locate the three wires by measuring the distances between these three and some known reference points. With these lengths, one can easily calculate the $x$ and the $y$ coordinates of each wire relative to one of the reference points, and relative to some chosen directions for the $x$ and the $y$ axes.

It appears that only two such points are needed. One point serves as the origin of the Cartesian system of coordinates, and the other shows the direction of, e.g., the $x$ axis. Hence, it would be wise to place two sets of induction coils (or Hall sensors) at two points in the vicinity of the wires, and locate the wires relative to these two.

Using a laser-beam-based device, one can measure the distances between the two reference points-at which the induction coils (or Hall sensors) are placed—and the three wires to a reasonable accuracy. In the following analysis we assume that these lengths have been measured on the installation of the induction coils, and that these lengths are determined accurately enough. We also assume that the distance between the two reference points is known precisely enough.

Relative to the chosen system of coordinates, the measured lengths and the coordinates of the wires are defined according to Figure 2. In this figure, we have also illustrated the magnetic field vectors corresponding to the field generated by the current $I_{1}(t)$ at points $\mathrm{P} 1$ and P2, $\boldsymbol{B}_{11}$ and $\boldsymbol{B}_{12}$, respectively. Similarly, we have illustrated the magnetic field generated by the second current, $I_{2}(t)$, at the two points, $\boldsymbol{B}_{21}$ and $\boldsymbol{B}_{22}$, and the field produced by the third current, $I_{3}(t)$, at points P1 and P2, $\boldsymbol{B}_{31}$ and $\boldsymbol{B}_{32}$, respectively. The vectors are drawn by 


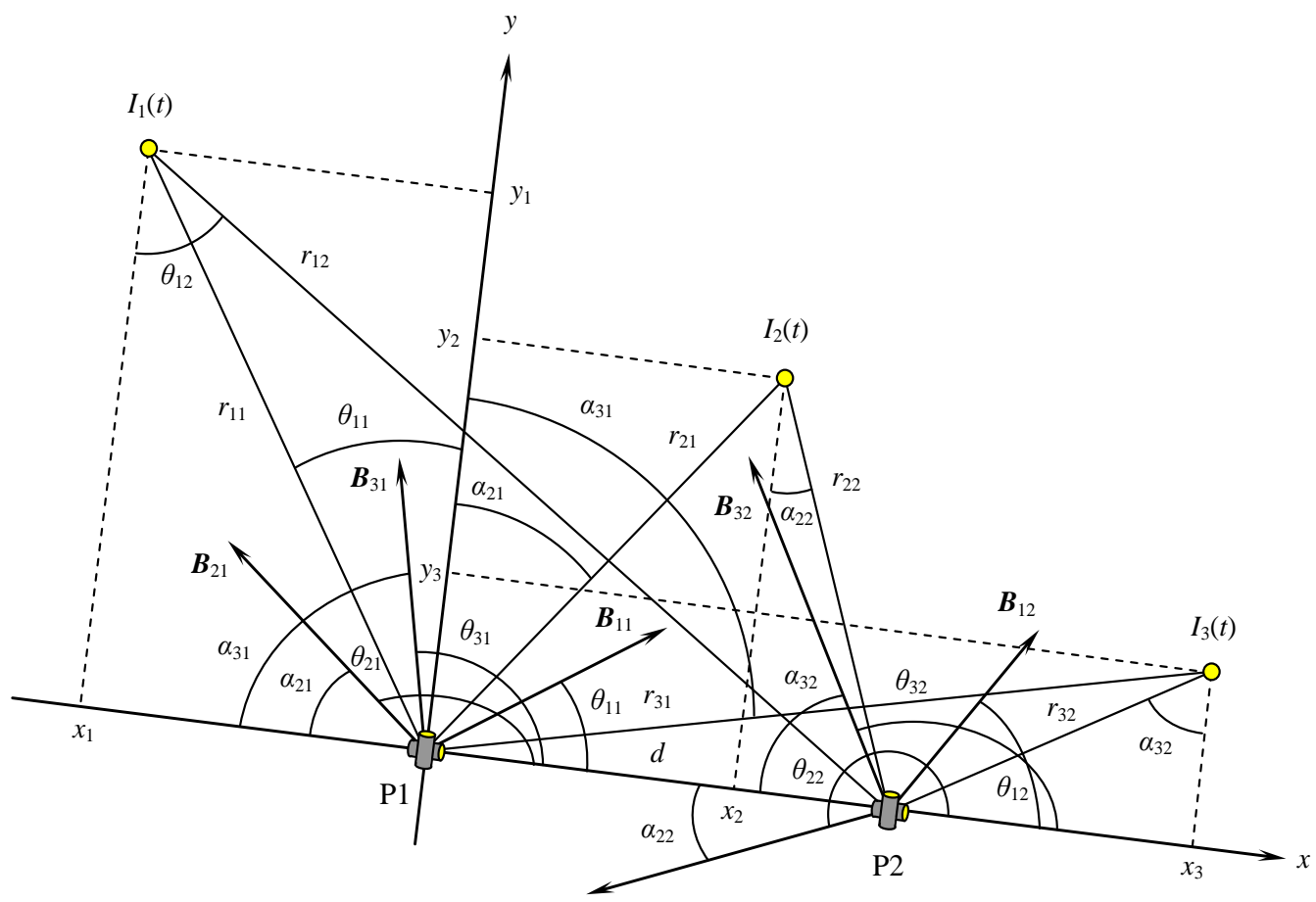

$\boldsymbol{B}_{22}$

Figure 2. The two sets of induction coils measure the induced emfs generated by the three sinusoidally oscillating currents.

assuming, as an example which does not restrict the generality of our analysis, that $t=0$ and that the currents are given by:

$$
\left\{\begin{array}{l}
I_{1}(t)=\hat{I}_{1} \cos \left(\omega t-\varphi_{1}\right) \\
I_{2}(t)=\hat{I}_{2} \cos \left(\omega t-\varphi_{2}\right) ; \\
I_{3}(t)=\hat{I}_{3} \cos \left(\omega t-\varphi_{3}\right)
\end{array} ;\left\{\begin{array}{l}
\varphi_{1}=0^{\circ} \\
\varphi_{2}=120^{\circ} \\
\varphi_{3}=240^{\circ}
\end{array}\right.\right.
$$

The figure was drawn somewhat slanted to remind the reader about the fact that the $x$ axis does not necessarily have to be horizontal; it only needs to point along a straight line passing through the two reference points. In our analysis, we assume that all the wires are parallel to each other, and that they point along the $z$ axis.

For a case of this kind, we need to use two induction coils at both reference points. One of these is directed along the $x$ axis, while the other points along the $y$ axis.

If the perpendicular distances $r_{11}, r_{12}, r_{21}, r_{22}, r_{31}$ and $r_{32}$ are measured during installation of the induction coils, for instance by using laser beam, one can determine the coordinates of the three wires by applying the Law of Cosines, see Figure 3:

$$
\left\{\begin{array}{l}
r_{11}^{2}=r_{12}^{2}+d^{2}-2 r_{12} d \cos \beta_{1} \\
r_{21}^{2}=r_{22}^{2}+d^{2}-2 r_{22} d \cos \beta_{2} \\
r_{31}^{2}=r_{32}^{2}+d^{2}-2 r_{32} d \cos \beta_{3}
\end{array}\right.
$$

These yield:

$$
\begin{aligned}
& \beta_{1}=\cos ^{-1}\left(\frac{r_{12}^{2}+d^{2}-r_{11}^{2}}{2 r_{12} d}\right) ;\left\{\begin{array}{l}
x_{1}=d-r_{12} \cos \beta_{1} \\
y_{1}=r_{12} \sin \beta_{1}
\end{array}\right. \\
& \beta_{2}=\cos ^{-1}\left(\frac{r_{22}^{2}+d^{2}-r_{21}^{2}}{2 r_{22} d}\right) ;\left\{\begin{array}{l}
x_{2}=d-r_{22} \cos \beta_{2} \\
y_{2}=r_{22} \sin \beta_{2}
\end{array}\right.
\end{aligned}
$$




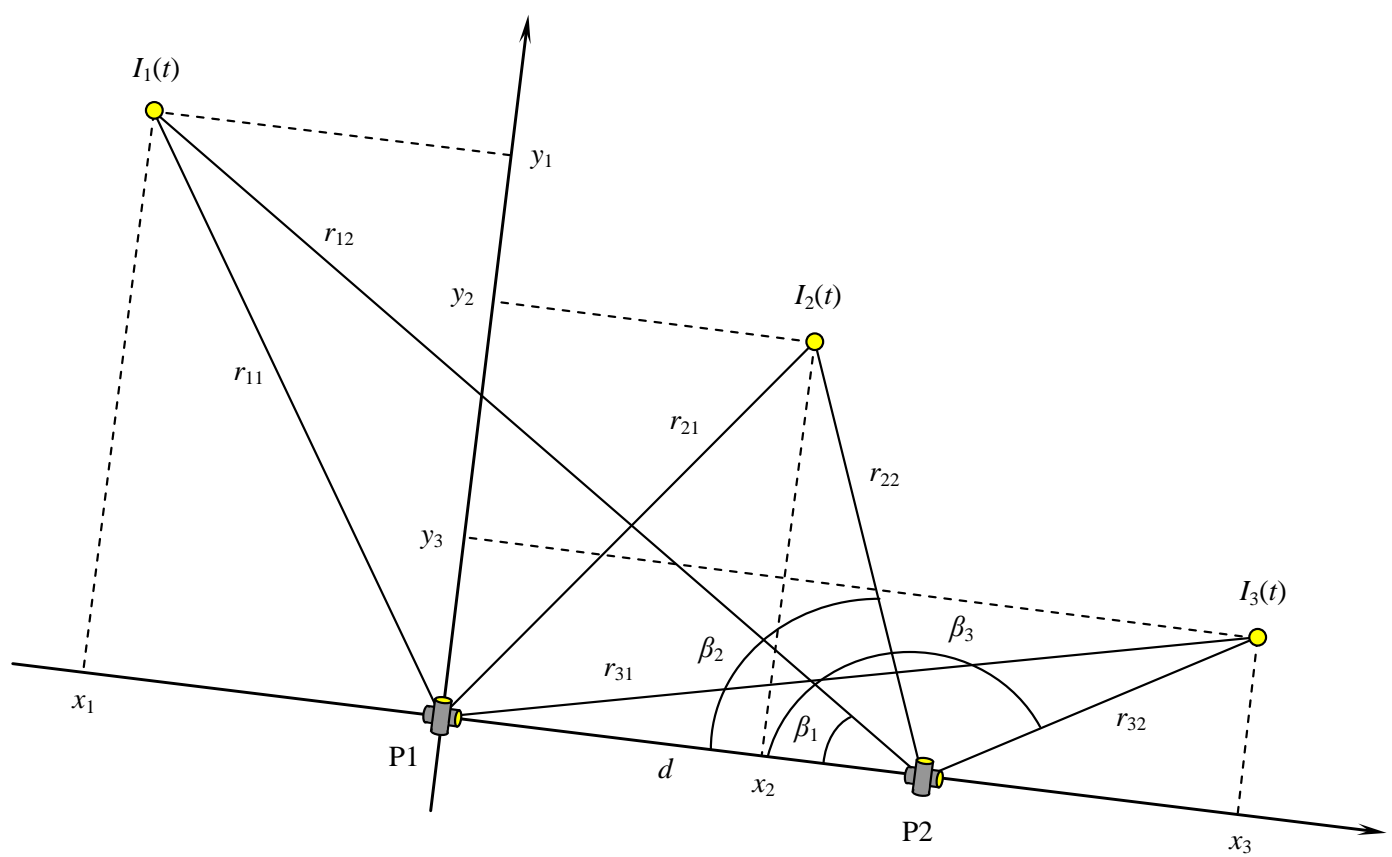

Figure 3. A simplified illustration of the geometry.

$$
\beta_{3}=\cos ^{-1}\left(\frac{r_{32}^{2}+d^{2}-r_{31}^{2}}{2 r_{32} d}\right) ;\left\{\begin{array}{l}
x_{3}=d-r_{32} \cos \beta_{3} \\
y_{3}=r_{32} \sin \beta_{3}
\end{array}\right.
$$

The emf induced in the $x$-directed coil at point P1 can be given in terms of the three currents, the calculated wire coordinates and the angles defined in Figure 2:

$$
\begin{aligned}
\varepsilon_{1 x}(t) & =-N_{1 x} \frac{\mathrm{d}}{\mathrm{d} t}\left\{\boldsymbol{A}_{1 x} \cdot \boldsymbol{B}_{11}(t)+\boldsymbol{A}_{1 x} \cdot \boldsymbol{B}_{21}(t)+\boldsymbol{A}_{1 x} \cdot \boldsymbol{B}_{31}(t)\right\} \\
& =-N_{1 x} \frac{\mathrm{d}}{\mathrm{d} t}\left\{\pi r_{1 x}^{2}\left[B_{11}(t) \cos \theta_{11}+B_{21}(t) \cos \theta_{21}+B_{31}(t) \cos \theta_{31}\right]\right\} \\
& =-N_{1 x} \pi r_{1 x}^{2} \frac{\mathrm{d}}{\mathrm{d} t}\left\{\frac{\mu_{0} I_{1}(t)}{2 \pi r_{11}} \cos \theta_{11}+\frac{\mu_{0} I_{2}(t)}{2 \pi r_{21}} \cos \theta_{21}+\frac{\mu_{0} I_{3}(t)}{2 \pi r_{31}} \operatorname{co} \theta_{31}\right\}
\end{aligned}
$$

In this expression, $N_{1 x}$ is the number of turns of wire in the induction coil oriented along the $x$ axis and placed at P1; $r_{1 x}$ is the radius of this coil. According to Figure 2, the cosines of the angles in this result can be given as:

$$
\cos \theta_{11}=\frac{y_{1}}{r_{11}} ; \cos \theta_{21}=-\frac{y_{2}}{r_{21}} ; \cos \theta_{31}=-\frac{y_{3}}{r_{31}}
$$

Therefore, we can give the emf induced in the $x$-directed coil at P1 as:

$$
\begin{aligned}
\varepsilon_{1 x}(t) & =-\frac{N_{1 x} r_{1 x}^{2} \mu_{0}}{2} \frac{\mathrm{d}}{\mathrm{d} t}\left\{\frac{\hat{I}_{1} \cos \left(\omega t-\varphi_{1}\right)}{r_{11}} \frac{y_{1}}{r_{11}}+\frac{\hat{I}_{2} \cos \left(\omega t-\varphi_{2}\right)}{r_{21}}\left(-\frac{y_{2}}{r_{21}}\right)+\frac{\hat{I}_{3} \cos \left(\omega t-\varphi_{3}\right)}{r_{31}}\left(-\frac{y_{3}}{r_{31}}\right)\right\} \\
& =\frac{N_{1 x} r_{1 x}^{2} \mu_{0} \omega}{2}\left\{\frac{y_{1}}{r_{11}^{2}} \hat{I}_{1} \sin \left(\omega t-\varphi_{1}\right)-\frac{y_{2}}{r_{21}^{2}} \hat{I}_{2} \sin \left(\omega t-\varphi_{2}\right)-\frac{y_{3}}{r_{31}^{2}} \hat{I}_{3} \sin \left(\omega t-\varphi_{3}\right)\right\}
\end{aligned}
$$

Similarly, we can show that the emf induced in the $x$-directed coil at P2 is given by:

$$
\varepsilon_{2 x}(t)=\frac{N_{2 x} r_{2 x}^{2} \mu_{0} \omega}{2}\left\{\frac{y_{1}}{r_{12}^{2}} \hat{I}_{1} \sin \left(\omega t-\varphi_{1}\right)-\frac{y_{2}}{r_{22}^{2}} \hat{I}_{2} \sin \left(\omega t-\varphi_{2}\right)-\frac{y_{3}}{r_{32}^{2}} \hat{I}_{3} \sin \left(\omega t-\varphi_{3}\right)\right\}
$$

Here, $N_{2 x}$ is the number of turns of wire in the second induction coil oriented along the $x$ axis and placed at P2; 
$r_{2 x}$ is the radius of this coil.

We can express these results more simply if we rewrite them in terms of factors $k_{1}, k_{2}, \cdots, k_{6}$ as follows:

$$
\begin{aligned}
& \varepsilon_{1 x}(t)=k_{1} \hat{I}_{1} \sin \left(\omega t-\varphi_{1}\right)+k_{2} \hat{I}_{2} \sin \left(\omega t-\varphi_{2}\right)+k_{3} \hat{I}_{3} \sin \left(\omega t-\varphi_{3}\right) ;\left\{\begin{array}{l}
k_{1}=\frac{N_{1 x} r_{1 x}^{2} \mu_{0} \omega}{2} \frac{y_{1}}{r_{11}^{2}} \\
k_{2}=-\frac{N_{1 x} r_{1 x}^{2} \mu_{0} \omega}{2} \frac{y_{2}}{r_{21}^{2}} \\
k_{3}=-\frac{N_{1 x} r_{1 x}^{2} \mu_{0} \omega}{2} \frac{y_{3}}{r_{31}^{2}}
\end{array}\right. \\
& \varepsilon_{2 x}(t)=k_{4} \hat{I}_{1} \sin \left(\omega t-\varphi_{1}\right)+k_{5} \hat{I}_{2} \sin \left(\omega t-\varphi_{2}\right)+k_{6} \hat{I}_{3} \sin \left(\omega t-\varphi_{3}\right) ;\left\{\begin{array}{l}
k_{4}=\frac{N_{2 x} r_{2 x}^{2} \mu_{0} \omega}{2} \frac{y_{1}}{r_{12}^{2}} \\
k_{5}=-\frac{N_{2 x} r_{2 x}^{2} \mu_{0} \omega}{2} \frac{y_{2}}{r_{22}^{2}} \\
k_{6}=-\frac{N_{2 x} r_{2 x}^{2} \mu_{0} \omega}{2} \frac{y_{3}}{r_{32}^{2}}
\end{array}\right.
\end{aligned}
$$

The difference in voltage across the two $x$-directed coils can be given as:

$$
\begin{aligned}
\Delta \varepsilon_{x}(t) & =\varepsilon_{2 x}(t)-\varepsilon_{1 x}(t)=\frac{\mu_{0} \omega y_{1} \hat{I}_{1} \sin \left(\omega t-\varphi_{1}\right)}{2}\left\{\frac{N_{2 x} r_{2 x}^{2}}{r_{12}^{2}}-\frac{N_{1 x} r_{1 x}^{2}}{r_{11}^{2}}\right\} \\
& +\frac{\mu_{0} \omega y_{2} \hat{I}_{2} \sin \left(\omega t-\varphi_{1}\right)}{2}\left\{-\frac{N_{2 x} r_{2 x}^{2}}{r_{22}^{2}}+\frac{N_{1 x} r_{1 x}^{2}}{r_{21}^{2}}\right\}+\frac{\mu_{0} \omega \hat{y}_{3} \hat{I}_{3} \sin \left(\omega t-\varphi_{1}\right)}{2}\left\{-\frac{N_{2 x} r_{2 x}^{2}}{r_{32}^{2}}+\frac{N_{1 x} r_{1 x}^{2}}{r_{31}^{2}}\right\}
\end{aligned}
$$

If we call the factors that multiply the current amplitudes as $k_{7}, k_{8}$ and $k_{9}$, this result can be given in a shorter form as:

$$
\Delta \varepsilon_{x}(t)=k_{7} \hat{I}_{1} \sin \left(\omega t-\varphi_{1}\right)+k_{8} \hat{I}_{2} \sin \left(\omega t-\varphi_{2}\right)+k_{9} \hat{I}_{3} \sin \left(\omega t-\varphi_{3}\right) ;\left\{\begin{array}{l}
k_{7}=\frac{\mu_{0} \omega y_{1}}{2}\left(\frac{N_{2 x} r_{2 x}^{2}}{r_{12}^{2}}-\frac{N_{1 x} r_{1 x}^{2}}{r_{11}^{2}}\right) \\
k_{8}=\frac{\mu_{0} \omega y_{2}}{2}\left(\frac{N_{1 x} r_{1 x}^{2}}{r_{21}^{2}}-\frac{N_{2 x} r_{2 x}^{2}}{r_{22}^{2}}\right) \\
k_{9}=\frac{\mu_{0} \omega y_{3}}{2}\left(\frac{N_{1 x} r_{1 x}^{2}}{r_{31}^{2}}-\frac{N_{2 x} r_{2 x}^{2}}{r_{32}^{2}}\right)
\end{array}\right.
$$

Note that the current terms in this result are the actual currents flowing in the three wires, except for the phase difference of $\frac{\pi}{2}$ radians. This means that we can find out the time-dependent currents $I_{1}(t), I_{2}(t)$ and $I_{3}(t)$ —with the phase difference of $\frac{\pi}{2}$ —by solving the following matrix equation:

$$
\left(\begin{array}{lll}
k_{1} & k_{2} & k_{3} \\
k_{4} & k_{5} & k_{6} \\
k_{7} & k_{8} & k_{9}
\end{array}\right)\left(\begin{array}{l}
I_{1}(t)^{\prime} \\
I_{2}(t)^{\prime} \\
I_{3}(t)^{\prime}
\end{array}\right)=\left(\begin{array}{c}
\varepsilon_{1 x}(t) \\
\varepsilon_{2 x}(t) \\
\Delta \varepsilon_{x}(t)
\end{array}\right)
$$

Here, the primed symbols refer to the currents, which differ from the real ones by the phase difference of $\frac{\pi}{2}$ rad.

Following a similar approach, one can derive the following result for the $y$-directed coil at P1: 


$$
\begin{aligned}
\varepsilon_{1 y}(t) & =-N_{1 y} \frac{\mathrm{d}}{\mathrm{d} t}\left\{\boldsymbol{A}_{1 y} \cdot \boldsymbol{B}_{11}(t)+\boldsymbol{A}_{1 y} \cdot \boldsymbol{B}_{21}(t)+\boldsymbol{A}_{1 y} \cdot \boldsymbol{B}_{31}(t)\right\} \\
& =-N_{1 y} \frac{\mathrm{d}}{\mathrm{d} t}\left\{\pi r_{1 y}^{2}\left[B_{11}(t) \cos \left(\frac{\pi}{2}-\theta_{11}\right)+B_{21}(t) \cos \left(\theta_{21}-\frac{\pi}{2}\right)+B_{31}(t) \cos \left(\theta_{31}-\frac{\pi}{2}\right)\right]\right\}
\end{aligned}
$$

In this expression, $N_{1 y}$ is the number of turns of wire in the induction coil oriented along the $y$ axis and placed at P1; $r_{1 y}$ is the radius of this coil. According to Figure 2 and the fundamental rules of trigonometry, the trigonometric terms in this result can be given as:

$$
\begin{aligned}
& \cos \left(\frac{\pi}{2}-\theta_{11}\right)=\cos \left(\theta_{11}-\frac{\pi}{2}\right)=\sin \theta_{11}=\frac{x_{1}}{r_{11}} \\
& \cos \left(\theta_{21}-\frac{\pi}{2}\right)=\sin \theta_{21}=-\sin \left(2 \pi-\theta_{21}\right)=-\sin \alpha_{21}=-\frac{x_{2}}{r_{21}} \\
& \cos \left(\theta_{31}-\frac{\pi}{2}\right)=\sin \theta_{31}=-\sin \left(2 \pi-\theta_{31}\right)=-\sin \alpha_{31}=-\frac{x_{3}}{r_{31}}
\end{aligned}
$$

These results yield:

$$
\begin{aligned}
\varepsilon_{1 y}(t) & =-N_{1 y} \pi r_{1 y}^{2} \frac{\mathrm{d}}{\mathrm{d} t}\left\{\frac{\mu_{0} I_{1}(t)}{2 \pi r_{11}} \frac{x_{1}}{r_{11}}-\frac{\mu_{0} I_{2}(t)}{2 \pi r_{21}} \frac{x_{2}}{r_{21}}-\frac{\mu_{0} I_{3}(t)}{2 \pi r_{31}} \frac{x_{3}}{r_{31}}\right\} \\
& =-\frac{N_{1 y} r_{1 y}^{2} \mu_{0}}{2} \frac{\mathrm{d}}{\mathrm{d} t}\left\{\frac{x_{1}}{r_{11}^{2}} \hat{I}_{1} \cos \left(\omega t-\varphi_{1}\right)-\frac{x_{2}}{r_{21}^{2}} \hat{I}_{2} \cos \left(\omega t-\varphi_{2}\right)-\frac{x_{3}}{r_{31}^{2}} \hat{I}_{3} \cos \left(\omega t-\varphi_{3}\right)\right\} \\
& =\frac{N_{1 y} r_{1 y}^{2} \mu_{0} \omega}{2}\left\{\frac{x_{1}}{r_{11}^{2}} \hat{I}_{1} \sin \left(\omega t-\varphi_{1}\right)-\frac{x_{2}}{r_{21}^{2}} \hat{I}_{2} \sin \left(\omega t-\varphi_{2}\right)-\frac{x_{3}}{r_{31}^{2}} \hat{I}_{3} \sin \left(\omega t-\varphi_{3}\right)\right\}
\end{aligned}
$$

Again, we can express this result more simply if we rewrite it in terms of factors $p_{1}, p_{2}$ and $p_{3}$ as follows:

$$
\varepsilon_{1 y}(t)=p_{1} \hat{I}_{1} \sin \left(\omega t-\varphi_{1}\right)+p_{2} \hat{I}_{2} \sin \left(\omega t-\varphi_{2}\right)+p_{3} \hat{I}_{3} \sin \left(\omega t-\varphi_{3}\right) ;\left\{\begin{array}{c}
p_{1}=\frac{N_{1 y} r_{1 y}^{2} \mu_{0} \omega}{2} \frac{x_{1}}{r_{11}^{2}} \\
p_{2}=-\frac{N_{1 y} r_{1 y}^{2} \mu_{0} \omega}{2} \frac{x_{2}}{r_{21}^{2}} \\
p_{3}=-\frac{N_{1 y} r_{1 y}^{2} \mu_{0} \omega}{2} \frac{x_{3}}{r_{31}^{2}}
\end{array}\right.
$$

Similarly, we can show that the emf induced in the $y$-directed coil at P2 is given by:

$$
\begin{aligned}
\varepsilon_{2 y}(t) & =-N_{2 y} \frac{\mathrm{d}}{\mathrm{d} t}\left\{\boldsymbol{A}_{2 y} \cdot \boldsymbol{B}_{12}(t)+\boldsymbol{A}_{2 y} \cdot \boldsymbol{B}_{22}(t)+\boldsymbol{A}_{2 y} \cdot \boldsymbol{B}_{32}(t)\right\} \\
& =-N_{2 y} \pi r_{2 y}^{2} \frac{\mathrm{d}}{\mathrm{d} t}\left\{\frac{\mu_{0} I_{1}(t)}{2 \pi r_{12}} \cos \left(\frac{\pi}{2}-\theta_{12}\right)+\frac{\mu_{0} I_{2}(t)}{2 \pi r_{22}} \cos \left(\theta_{22}-\frac{\pi}{2}\right)+\frac{\mu_{0} I_{3}(t)}{2 \pi r_{32}} \cos \left(\theta_{32}-\frac{\pi}{2}\right)\right\}
\end{aligned}
$$

Here, $N_{2 y}$ is the number of turns of wire in the induction coil oriented along the $y$ axis and placed at P2; $r_{2 y}$ is the radius of this coil. According to laws of trigonometry and Figure 2, the cosines of the angles in this result can be given as:

$$
\begin{aligned}
& \cos \left(\frac{\pi}{2}-\theta_{12}\right)=\sin \theta_{12}=\frac{d-x_{1}}{r_{12}} \\
& \cos \left(\theta_{22}-\frac{\pi}{2}\right)=\cos \left(\frac{\pi}{2}-\theta_{22}\right)=\sin \theta_{22}=-\sin \left(\theta_{22}-\pi\right)=-\sin \alpha_{22}=-\frac{d-x_{2}}{r_{22}} \\
& \cos \left(\theta_{32}-\frac{\pi}{2}\right)=\cos \left(\frac{\pi}{2}-\theta_{32}\right)=\sin \theta_{32}=\sin \left(\pi-\theta_{32}\right)=\sin \alpha_{32}=\frac{x_{3}-d}{r_{32}}
\end{aligned}
$$


Thus, we can give the emf induced in the $y$-directed coil at P2 as:

$$
\begin{aligned}
\varepsilon_{2 y}(t) & =-\frac{N_{2 y} r_{2 y}^{2} \mu_{0}}{2} \frac{\mathrm{d}}{\mathrm{d} t}\left\{\frac{\hat{I}_{1} \cos \left(\omega t-\varphi_{1}\right)}{r_{12}} \frac{d-x_{1}}{r_{12}}+\frac{\hat{I}_{2} \cos \left(\omega t-\varphi_{2}\right)}{r_{22}}\left(-\frac{d-x_{2}}{r_{22}}\right)+\frac{\hat{I}_{3} \cos \left(\omega t-\varphi_{3}\right)}{r_{32}} \frac{x_{3}-d}{r_{32}}\right\} \\
& =-\frac{N_{2 y} r_{2 y}^{2} \mu_{0} \omega}{2}\left\{-\frac{d-x_{1}}{r_{12}^{2}} \hat{I}_{1} \sin \left(\omega t-\varphi_{1}\right)+\frac{d-x_{2}}{r_{22}^{2}} \hat{I}_{2} \sin \left(\omega t-\varphi_{2}\right)+\frac{d-x_{3}}{r_{32}^{2}} \hat{I}_{3} \sin \left(\omega t-\varphi_{3}\right)\right\} \\
& =\frac{N_{2 y} r_{2 y}^{2} \mu_{0} \omega}{2}\left\{\frac{d-x_{1}}{r_{12}^{2}} \hat{I}_{1} \sin \left(\omega t-\varphi_{1}\right)-\frac{d-x_{2}}{r_{22}^{2}} \hat{I}_{2} \sin \left(\omega t-\varphi_{2}\right)-\frac{d-x_{3}}{r_{32}^{2}} \hat{I}_{3} \sin \left(\omega t-\varphi_{3}\right)\right\}
\end{aligned}
$$

Let us rewrite this result in terms of factors $p_{4}, p_{5}$ and $p_{6}$ :

$$
\varepsilon_{2 y}(t)=p_{4} \hat{I}_{1} \sin \left(\omega t-\varphi_{1}\right)+p_{5} \hat{I}_{2} \sin \left(\omega t-\varphi_{2}\right)+p_{6} \hat{I}_{3} \sin \left(\omega t-\varphi_{3}\right) ;\left\{\begin{array}{l}
p_{4}=\frac{N_{2 y} r_{2 y}^{2} \mu_{0} \omega}{2} \frac{d-x_{1}}{r_{12}^{2}} \\
p_{5}=-\frac{N_{2 y} r_{2 y}^{2} \mu_{0} \omega \frac{d-x_{2}}{2}}{r_{22}^{2}} \\
p_{6}=-\frac{N_{2 y} r_{2 y}^{2} \mu_{0} \omega \frac{d-x_{3}}{2}}{r_{32}^{2}}
\end{array}\right.
$$

The difference in voltage across the two $y$-directed coils can be given as:

$$
\begin{aligned}
\Delta \varepsilon_{y}(t) & =\varepsilon_{2 y}(t)-\varepsilon_{1 y}(t)=\frac{\mu_{0} \omega \hat{I}_{1} \sin \left(\omega t-\varphi_{1}\right)}{2}\left\{N_{2 y} r_{2 y}^{2} \frac{d-x_{1}}{r_{12}^{2}}-N_{1 y} r_{1 y}^{2} \frac{x_{1}}{r_{11}^{2}}\right\} \\
& +\frac{\mu_{0} \omega \hat{I}_{2} \sin \left(\omega t-\varphi_{2}\right)}{2}\left\{N_{2 y} r_{2 y}^{2} \frac{x_{2}-d}{r_{22}^{2}}+N_{1 y} r_{1 y}^{2} \frac{x_{2}}{r_{21}^{2}}\right\}+\frac{\mu_{0} \omega \hat{I}_{3} \sin \left(\omega t-\varphi_{3}\right)}{2}\left\{N_{2 y} r_{2 y}^{2} \frac{x_{3}-d}{r_{32}^{2}}+N_{1 y} r_{1 y}^{2} \frac{x_{3}}{r_{31}^{2}}\right\}
\end{aligned}
$$

This result can be given in a shorter form as:

$$
\Delta \varepsilon_{y}(t)=p_{7} \hat{I}_{1} \sin \left(\omega t-\varphi_{1}\right)+p_{8} \hat{I}_{2} \sin \left(\omega t-\varphi_{2}\right)+p_{9} \hat{I}_{3} \sin \left(\omega t-\varphi_{3}\right) ;\left\{\begin{array}{l}
p_{7}=\frac{\mu_{0} \omega}{2}\left(N_{2 y} r_{2 y}^{2} \frac{d-x_{1}}{r_{12}^{2}}-N_{1 y} r_{1 y}^{2} \frac{x_{1}}{r_{11}^{2}}\right) \\
p_{8}=\frac{\mu_{0} \omega}{2}\left(N_{2 y} r_{2 y}^{2} \frac{x_{2}-d}{r_{22}^{2}}+N_{1 y} r_{1 y}^{2} \frac{x_{2}}{r_{21}^{2}}\right) \\
p_{9}=\frac{\mu_{0} \omega}{2}\left(N_{2 y} r_{2 y}^{2} \frac{x_{3}-d}{r_{32}^{2}}+N_{1 y} r_{1 y}^{2} \frac{x_{3}}{r_{31}^{2}}\right)
\end{array}\right.
$$

Note that the current terms in this result are the actual currents flowing in the three wires, except for the phase difference of $\frac{\pi}{2}$ radians.

We can find out the time-dependent currents $I_{1}(t), I_{2}(t)$ and $I_{3}(t)$-with the phase difference of $\frac{\pi}{2}-$ by solving the following matrix equation:

$$
\left(\begin{array}{lll}
p_{1} & p_{2} & p_{3} \\
p_{4} & p_{5} & p_{6} \\
p_{7} & p_{8} & p_{9}
\end{array}\right)\left(\begin{array}{c}
I_{1}(t)^{\prime} \\
I_{2}(t)^{\prime} \\
I_{3}(t)^{\prime}
\end{array}\right)=\left(\begin{array}{c}
\varepsilon_{1 y}(t) \\
\varepsilon_{2 y}(t) \\
\Delta \varepsilon_{y}(t)
\end{array}\right)
$$

Here, the primed symbols refer to the currents, which differ from the real ones by the phase difference of $\frac{\pi}{2}$ rad. 


\section{Applying the Results}

The device described in this article could be used so that both sensors, at P1 and at P2, measure the induced voltage at least once in every millisecond. One measurement sequence could last for about 20 periods of current oscillations, and such sequences could be measured once in every 10 minutes, for example. The collected data could then be transmitted wirelessly to a remote receiver. The received data could be analyzed in the office using software based on the matrix equations derived in this article.

The software could calculate the currents $I_{1}(t)^{\prime}, I_{2}(t)^{\prime}$ and $I_{3}(t)^{\prime}$ with both of the given matrix equations. After this, one could compare the results with each other, and if they were close to each other within reasonable accuracy, the final results could be found by calculating the average values of the two. Finally, the actual currents, $I_{1}(t), I_{2}(t)$ and $I_{3}(t)$ would be obtained by shifting the sinusoidal functions of time by $\frac{\pi}{2}$ rad.

The device should be equipped with two additional induction coils along the device's $z$ axis. On installation, one should make sure that the voltage in these coils is zero. This ensures that the two sets of perpendicular coils at $\mathrm{P} 1$ and at $\mathrm{P} 2$ are in the $x y$ plane perpendicular to the three current-carrying wires.

\section{Conclusion}

Our analysis indicates that it is possible to build a device that can determine three sinusoidally oscillating currents as functions of time from a reasonable distance. The device can be installed safely to the measuring site without turning the power off in the transmission lines. The next logical step in our process would be to build a device explained in this article, and to make test runs with it and real transmission lines.

\section{References}

[1] Mäkinen, S. (2014) Toroidal Coil in Measuring Alternating Current at a Distance. Journal of Electromagnetic Analysis and Applications, 6, 367-371. http://dx.doi.org/10.4236/jemaa.2014.612037 